\title{
Designing a Deep Learning Hybrid Using CNN and Inception V3 Transfer Learning to Detect the Aggression Level of Deep Obsessive Compulsive Disorder in Children
}

\author{
Mukesh Madanan, Biju T.Sayed \\ Department of Computer Science, Dhofar University, Salalah, \\ Oman
}

Received: June 22, 2021. Revised: January 7, 2022. Accepted: January 24, 2022. Published: February 28, 2022

\begin{abstract}
The usage of Artificial intelligence in medical arena has proved to be a game changer in the detection and diagnosis of several medical conditions. In the current digital era, children with stressful medical issues are suffering from Deep Obsessive-Compulsive Disorder (DOCD). This kind of mental stress occurs in children because of the continuous usage of gadgets such as mobile phone, playing games using play stations, watching videos on tablets, etc. In most of the possibilities, single children are the ones affected with several obsessions such as stubborn activities, fighting for selfish priorities and so on. In medical terms, these kinds of complex behavioral changes are identified as DOCD. Genetic behaviors sometimes in a few group of children are also noticed as a modality difference. As symptoms are psychiatric impairment, such a child remains isolated, abnormal silence, being obsessive and repeating irrelevant words, high stress or anxiety. All medical challenges could be treated as healthcare research metrics and the gradual increase in DOCD disorder among children of this generation can be considered too. Early detection of DOCD is essential as it can help in early diagnosis but techniques to do so is unavailable currently. Deep learning-an artificial intelligence method can be utilized to detect DOCD, diagnose and treat it and bring about a positive character in children. Behavior changes in children can be classified and detected using transfer learning algorithms. In COVID-19 pandemic situation, 3\% of DOCD has increased to $10-15 \%$ as a disorder. This information is retrieved from children by monitoring negative activities, unusual behavior such as nail biting, removing spectacles and placing them in the wrong place, watching tablets, mobile phones and television for more hours. Using Convolutional Neural Networks (CNN), input such as MRI (Magnetic resonance Imaging) is used for experimenting the variations in behavior with the high dimension that are analyzed from the image dataset. Using Transfer Learning with Inception V3-, CNN generalization
\end{abstract}

of misophonia level can be statistically analyzed to avoid overfitting problems. By employing AI techniques, the aggression level can be predicted using data augmentation method with better accuracy and a low error rate than the existing systems. In the research it is observed that using the model employing Inception-V3 transfer learning CNN a better prediction of aggression levels can be achieved in comparison to the existing CNN model used.

Keywords- Convolutional Neural Networks, Deep Learning, Deep Obsessive-compulsive disorder, Genetic behaviors, InceptionV3-Transfer Learning, MRI.

\section{INTRODUCTION}

$\mathrm{D}$ EEP Obsessive Compulsive Disorder in people abbreviated as DOCD includes compulsive behaviors such as actions that are repetitive and unintentional [1]. The individuals affected by this disorder resist defying their excessive as well as unreasonable thoughts [4]. Symptoms related to the disorder include calculating things, hand washing frequently, obsessions such as checking locked doors repeatedly and so on [5]. Several other disorders such as anxiety, bipolar and risk of suicide are also associated with DOCD [2]. The factors of risk [6] related to it are events that include the stress along with abuse of the child. Other factors that may increase the possibility of this disorder are environmental and those with genetic ones [7]. Twins that are identical can be effected by the DOCD easily while comparing with the twins who are not identical [8].

The healthcare professionals make use of a handbook termed the Diagnostic and Statistical Manual of Mental Disorder (DSM) for diagnosing mental disorders. The practitioners in the medical field across the world use these instructions in DSM for diagnosing the presence of disorders in a particular individual. CBT termed as Cognitive Behavioral Therapy and ECT termed as Electroconvulsive Therapy are the techniques related to psychotherapy that are used to treat patients affected 
by DOCD [3]. Additionally, SSRI [9] termed as Selective Serotonin Reuptake Inhibitors are suggested often for the purpose of medications [10]. Clomipramine also provides medications similar to SSRI but generates side effects during treatment times. The treatment choice is based on the severity of the condition of the patient. Initially patients can be treated with CBT and later with SSRI. If the condition of the individual is severe, ECT can be recommended [11].

The research work done by Borjanka and team focussed on identifying the generic pattern related to the responsiveness of drug on the brain while using SSRI and the results of treatment while these SSRI are applied over an individual. Treatment is found to be effective where the response appears to be better [12]. In the region of the prefrontal cortex of the brain, the change in oxyhemoglobin appears for those who get effected by DOCD while observation is performed using infrared spectroscopy [13]. SSRI responders show good improvement while applying treatment compared to the others. Another medication called Tropisetron in combination of fluvoxamine also showed the improvement in treatment with people effected by this disorder [14]. SSRI in combination with any other psychotropic medications showed improvements in the symptoms of patients who were resistant to treatments [15].

The methods available in the computational field help in amplifying the evolution in the field of medicine as it contributes several discoveries for solving these puzzles that remain unsolved [16]. Several techniques are available in literature to find the existence of disease that seems to be complex such as cancer or illness related to psychiatric state of the person. The strategies in treatment can be personalized with the methods in computation that are advanced thereby creating a new way in the medical field. Personalized treatments help in providing customized ways of treatments to the patients for their diseases depending on the disease characteristics [17]. Data exploration in the field of biology with computational techniques gained their importance based on various studies made through them. People effected by depression can be identified and analyzed using machine learning methods [18]. GWAS termed as Genome Wide Association Study was performed in selective disorders including the patient depression, bipolar disorder in individuals, autism related disorders as well as schizophrenia. LASSO model helps in identifying parameters related to prediction in case of learning model. Training data can be constructed in SVM(Support Vector Machine) model [19].

The importance of machine learning methods is demonstrated for several software frameworks for review of genomic techniques [20]. Identification of genetic patterns in the patients suffering from cancer can be done using the models of machine learning on the datasets. Considering the schizophrenia patients [21], a classification model in combination with the framework for prediction was employed to find the treatment response. Another framework helped in diagnosing the gene markers effected by DOCD who are drug responsive. Individual profiles with gene expression were obtained and experiments were conducted for treatment with the SSRI. Non responders data is also found in the dataset used. Pre-processing of SNP termed as Single Nucleotide Polymorphism data is conducted with the initial phase followed by mRMR (minimum Redundancy Maximum Relevance) method for selecting the features based on filters. This eliminates the gene features that are found to be redundant in the dataset. The features that are remaining are sent into GRRF called as Guided Regularized Random Forest model. The main aim of this model is identification of specific subset of gene which plays a major role in the improvement of models for prediction.

By using training data, patterns were learned using the machine learning models and the evaluation of performance is with the help of test input. The above performance can be obtained by using the kcross-validation method where the " $k$ " can be chosen as 10 estimating the model skills in machine learning. CAE called as Contractive Auto Encoder is a deep learning approach which uses unsupervised modeling and is utilized in the final phase for creating classification models and also discriminating the samples. The features identified using GRRF are chosen as input and sent to the Contractive Auto Encoder. Data is analyzed to identify the logical representation of it which can be viewed as hidden weights and there is no need to eliminate the information that is relevant in this scheme. Performance comparison is performed by using the algorithms in machine learning.

In children the DOCD causes are limitless and to detect it by monitoring the aggressive nature may take time and treatment will be delayed. This is because the occurrence is detected only after the symptoms surface the children like aggressive behavior and rude attitude. In such a situation, there is a need of a system which would predict the nature of occurrence of DOCD by using advanced techniques. By studying the MRI report of children who previously had DOCD we could study the brain structure and conclude that this is the structure and changes that may happen when DOCD occurs. In such a situation we could use technology to study a group of DOCD children brains using image classification and compare it with children without DOCD. One way is to detect and measure the aggressive level and the connection between the brains of such child. Aggressiveness has effected the brain of children and this could be detected in MRI. Hence based on the thorough study of MRI of children we could in fact predict the occurrence of DOCD in a group of children by looking at the changes happening in the brain using MRI scans and also measure the level of aggressiveness.

Artificial intelligence methods could come in handy in the prediction of DOCD based on the MRI input. The paper aims to utilise the hybrid of $\mathrm{CNN}$ and Inception V3 Learning technique to detect and analyse the aggression level of DOCD especially in children.

The objective of the paper is as follows,

(i) To reduce blocks and the number of dimensions with less computation as well as mathematical calculation 
using Transfer learning -Inception V3 image classifier along with the $\mathrm{CNN}$ architecture.

(ii) To predict the aggression level on DOCD using transfer learning which can reduce the size of training to fit the proposed model as the best model using data augmentation.

iii) To attain higher accuracy in finding severity of aggression level on DOCD from softmax classifier and less error rate to improve the performance than the existing systems.

The paper is organized as follows: Section 2 addresses comparable works, Section 3 summarizes the proposed work, Section 4 delves into the findings, and Section 5 wraps up the paper.

\section{CLASSIFICATION}

Sequencing techniques with advanced features were done after completing the Human Genome project [22] by the year 2003. The generation of genomic data has seen a rapid optimal solution, as they are stochastic in nature. If a predictive model is used for computation of scores for a particular feature subset is called wrapper techniques. For this purpose, the statistical techniques used are correlation analysis, computation of mutual information and the tests based on values. On the other hand, manipulation of various techniques for selecting the feature subset for model construction is done in embedded technique. Few of the examples for this technique are LASSO and ridge methods.

Analysis for the written texts and applications based on DNA data also depend on the feature selection techniques which consists of several features of genes. Several outstanding contributions have been made in this field. In order to diagnose accurately [23], a framework was proposed in deep learning to predict the responsiveness towards the antidepressant drugs. This process was performed by medical practitioners with the help of gene data. Chronic hepatitis can be treated with the interferon and the evaluation process is performed with ANN using SNP information [24] over the

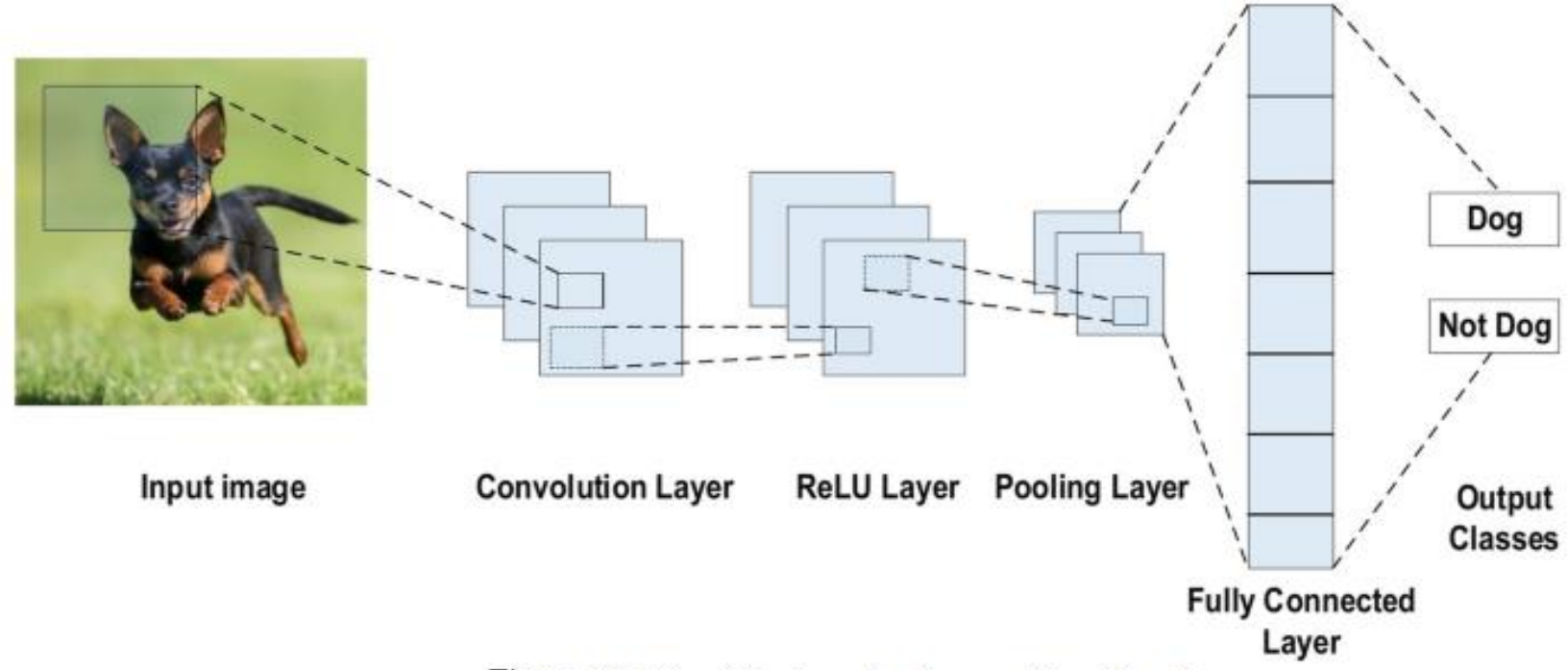

Fig 1: CNN architecture for Image Classification

increase in recent years. The devices used for storage were developed with more capacity and the systems used for computational purposes became more complex. These improvements led to the extraction of valuable information with these genomic data. Several fields including machine learning along with deep learning as well as artificial intelligence have obtained progress recently in an effective manner. Prediction in case of responsiveness to drugs, discovery of biomarkers and so on are the methods available for treatment that provide medications in a personalized manner.

In order to discover the biomarkers for diseases it is necessary to know the techniques available in existing systems. While considering a computational model, one of the crucial steps to consider is feature selection. For identification of subset with best features, various methods like filter or embedded or wrapper techniques can be utilized. By this, optimization problems can be solved which are difficult and do not have any relevant solution. These algorithms attain the individuals who were affected. A study comparing all the methods available for classification in case of prediction for this chronic syndrome using the SNP data is performed. Another approach used the SVM (Support Vector Machine) [25] for identification of responders as well as non responders for the usage of interferon as well as ribavirin together. Identifying genetic markers shows the treatment efficacy. The prediction model for treating depression for computation of medication efficacy of antidepressants is performed by utilizing stochastic gradient techniques [26]. The advantage of using CNN is that without any human supervision; the system can automatically detect vital information.

The treatment can be better understood by usage of biomarkers for prediction of antidepressants responsiveness in the patients [27]. Statistical methods are also used apart from these computational models to obtain better results. On application of SSRI, the response to treatment by individuals was predicted with usage of logistic regression [28]. Deep learning models produced a much lesser error rate while 
considering the data available as target [29]. RNA Eugene et al [30] was also used to perform analysis over genome data and also in finding their response for the antidepressants and also identification of biomarkers. Parameters used by CNN are much less while comparing with the network that is fully connected where the computation is performed using the convolution over smaller regions. The limitations in existing works lead to the development of the proposed work in detection of DOCD. Mukesh [31] stated for that the extraction of characteristics and classification of data from big image data sets is a major problem in microscopy image analysis. Artificial intelligence approaches have been widely utilized to predict outcomes in a variety of industries, including agriculture, economics, the stock market, and weather forecasting [31].

\section{A. Deep Learning}

One of the methods in artificial intelligence which helps in learning relationships using the data is called machine learning. Predictive models can be derived and there is no necessity for making assumptions regarding the mechanisms available that may be unknown. The workflow in machine learning includes harmonization of data, learning description, fitting of the model and the final stage is evaluation. The requirements used while construction of a system in machine learning is application of engineering methods in a careful manner and expertise in the domain for transformation of data in raw format to a format suitable for a classifier in order to identify the patterns in the given dataset. The conventional methods consist of processes in which input space is transformed linearly and are also not able to process the data available naturally into another form where it is raw.

When compared to machine learning, deep learning differs in the way in which the learning using raw data is done. Models which can do computation are made of layers used for processing in multiple levels depending on neural networks for learning of data representations. Deep learning differs from conventional ANNs(Artificial Neural Networks) with the hidden layers count, relations among them along with the input learning ability. There is a limitation of only 3 layers in case of conventional ANN and the training process is performed for obtaining the representations in supervised models.
Optimization can be done for performing only the particular task.

\section{1) Convolutional Neural network}

Of all the deep learning algorithms, the most frequently used one is the Convolutional Neural Network (CNN). The most significant feature that CNN has over its comparators is that it has the ability to identify significant characteristics and features. This is done in CNN without any supervision by humans. Inspired by the human brain neurons, CNNs have found its applications in face recognition applications, speech and voice recognitions systems and computer aided vision. In comparison to other rivals, the processing speed is up and the training procedure is much simpler for $\mathrm{CNN}$. In CNNs, the shared weights and local connections utilize the image signal to full extend and this is one of the major advantages of CNNs when compared to conventional neural networks. This factor is aids in speeding the processes in CNNs. CNNs are mostly used in classification problems as depicted in the Figure 1.

For the CNN architecture, the input image has height ' $\mathrm{m}$ ', width ' $\mathrm{m}$ ' and depth ' $\mathrm{r}$ '. In CNN, each convolutional layer is equipped with numerous kernels ' $\mathrm{k}$ ' with dimensions ' $\mathrm{n}$ ', ' $\mathrm{n}$ ' and ' $q$ ' whre ' $n$ ' is lesser ' $m$ ' and ' $q$ 'is lesser than or equal to ' $r$ '. All the kernels in CNN share similar parameters such as bias $\mathrm{b}^{\mathrm{k}}$ and weight $\mathrm{W}^{\mathrm{k}}$. $\mathrm{k}$-feature maps $\mathrm{h}^{\mathrm{k}}$ with dimensions $\mathrm{m}-\mathrm{n}$ 1 are generated. The dot product is calculated using the inputs and weights of the convolutional layers. The next step we apply the activation function to the output of the convolutional layer and obtain using equation (1)

$$
h^{k}=f\left(W^{k} * x+b^{k}\right)
$$

Next, each feature map in the sub-sampling layers is downsampled. This leads to a decrease in network parameters, which speeds up the training process and allows for the elimination of the overfitting problem. The pooling function (e.g. max or average) is applied to an adjacent area of size $\mathrm{p} \mathrm{x}$ $\mathrm{p}$, where $\mathrm{p}$ is the kernel size, for all feature maps. Finally, as in a conventional neural network, the FC layers receive the midand low-level features and construct the high-level abstraction, which reflects the last-stage layers. The final layer [e.g. support vector machines (SVMs) or softmax] is used to provide classification scores. Every score refers to the

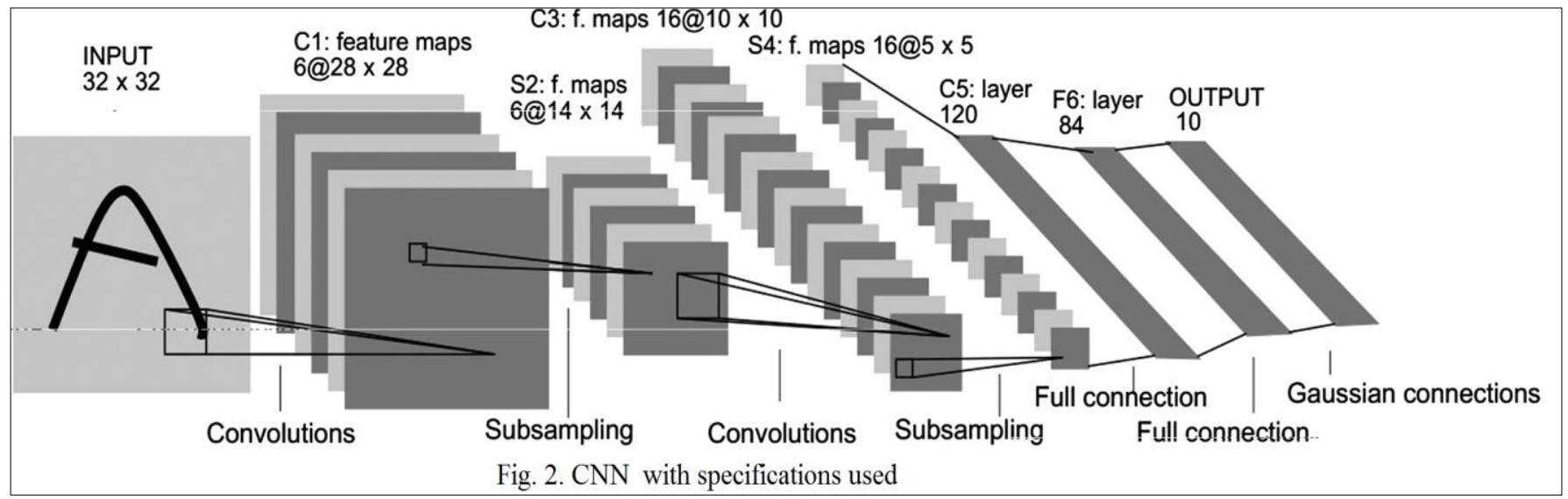


probability of something happening in a particular context.

There are multiple layers to the CNN architecture. Each layer of the CNN design, as well as its purpose, is explored in detail below.

a. Convolutional Layer: The convolutional layer is the most important component in $\mathrm{CNN}$ design. It is made up of a number of convolutional filters (so-called kernels). The output feature map is created by convoluting the input image with these filters, which are expressed as $\mathrm{N}$-dimensional metrics.

Kernel definition: The kernel is represented by a grid of discrete numbers or values. The kernel weight is the name given to each value. Random integers are assigned as kernel weights at the start of the CNN training process. Furthermore, the weights are initialized in a variety of ways. By altering these weights at each training session, the kernel learns to extract significant properties.

Convolutional Operation: First, the CNN input format is described. The vector format is the classic neural network's input, while the CNN's input is a multi-channeled picture. The gray-scale image format, for example, is single-channel, but the RGB image format is three-channeled. Let's look at a $4 \times 4$ image in grayscale with a $2 \times 2$ with a random weight initialization for the kernel. The kernel first goes across the entire image horizontally and vertically. The dot product of the input picture and the kernel is also calculated, with their respective values multiplied and then summed to generate a single scalar value. The operation is then repeated until no further sliding is feasible. It's worth noting that the output feature map is represented by the dot product values.

For the research, a two-dimensional (2-D) image of a single channel, each convolution kernel is a weight matrix, which can be a $3 \times 3$ or $5 \times 5$ matrix as shown in Figure 2 was considered.

Pooling Layer: The fundamental role of the pooling layer is to subsample the feature maps. These maps are made using convolutional operations. To put it another way, this strategy minimizes the size of large feature maps so that smaller feature maps can be created. At the same time, it preserves the bulk of the dominating information at each level of the pooling process (or features). Prior to the pooling method, the stride and kernel are both size-assigned, in the similar manner that the convolutional operation is. There are a multitude of pooling strategies to choose from in various pooling levels. Tree pooling, gated pooling, average pooling, min pooling, max pooling, global average pooling (GAP), and global max pooling are amongst the mechanisms which could be used. As demonstrated in Figure 3, the most well-known and extensively used pooling algorithms are max, min, and GAP pooling.

Tanh: It's similar to the sigmoid function in that it accepts real values as input, but the output is limited to a range of 1 to -1. It is mathematically formulated in

$$
f(x)_{\tanh }=\frac{e^{x}-e^{-x}}{e^{x}+e^{-x}}
$$

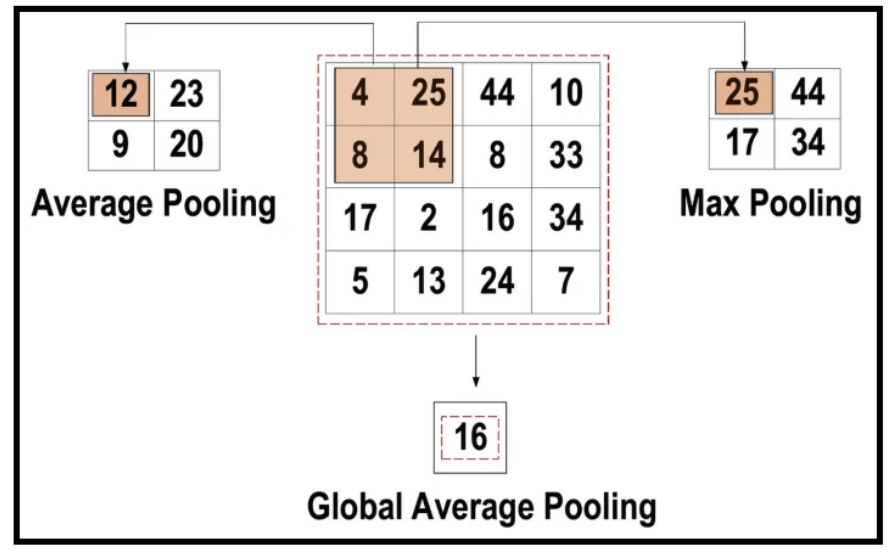

Fig 3. Pooling Algorithms

ReLU: In the context of CNN, ReLU is by far the most frequently used function. It converts the whole numbers in the input to positive numbers. ReLU has a significant edge over the others in terms of computational load. Its mathematical form is Eq. 4.

$$
f(x)_{R e L U}=\max (0, x)
$$

Leaky ReLU: Instead of the negative inputs being downscaled by the ReLU, this activation function ensures that they are never ignored. It's used to solve the Dying ReLU issue. Eq. 5 shows how a leaky ReLU can be represented mathematically. $f(x)_{\text {LeakyReLU }}=\left\{\begin{array}{ll}x, & \text { if } x>0 \\ m x, & x \leq 0\end{array}\right\}$

where $\mathrm{m}$ stands for the leak factor. It's usually set to an extremely low number, like 0.001 .

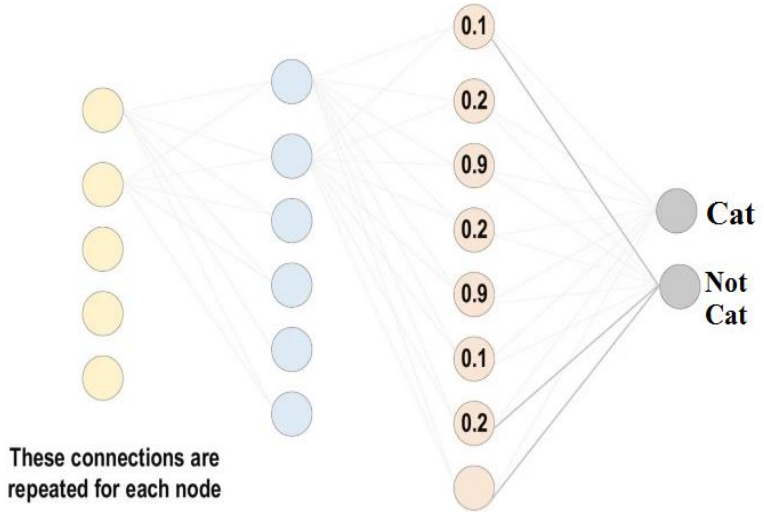

Fig 4. Fully Connected Layer 


\section{Inception v3 Model}

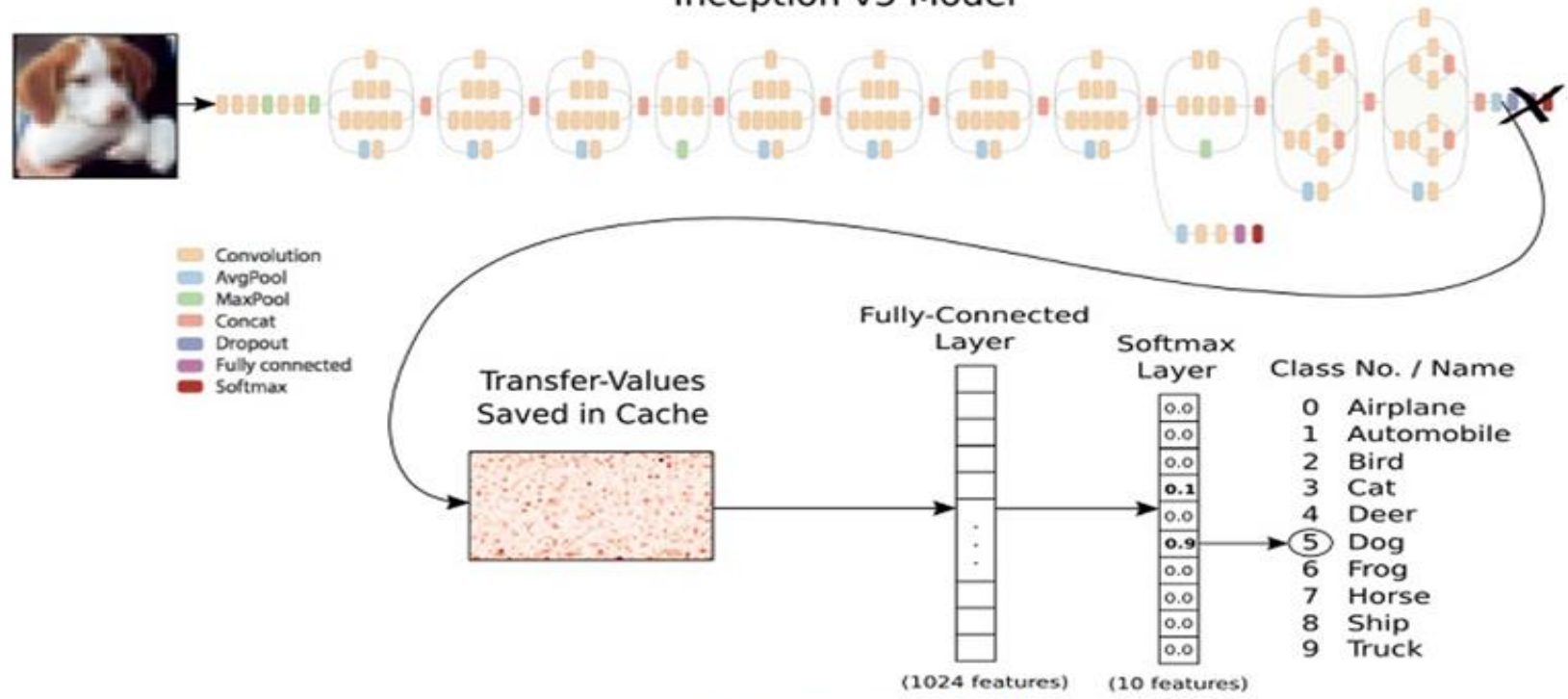

Fig 5. Inception V3 Model

Noisy ReLU: To make ReLU noisy, this function uses a

Gaussian distribution. Eq. 6 is a mathematical representation of it.

$$
f(x)_{\text {NoisyeLU }}=\max (x+Y), \text { with } Y \sim N(0, \sigma(x))
$$

Leaky ReLU: This is essentially the same as Parametric Linear Units. The primary distinction is that in this function, the leak factor is adjusted during the model training process. Eq. 7 is a mathematical representation of the parametric linear unit.
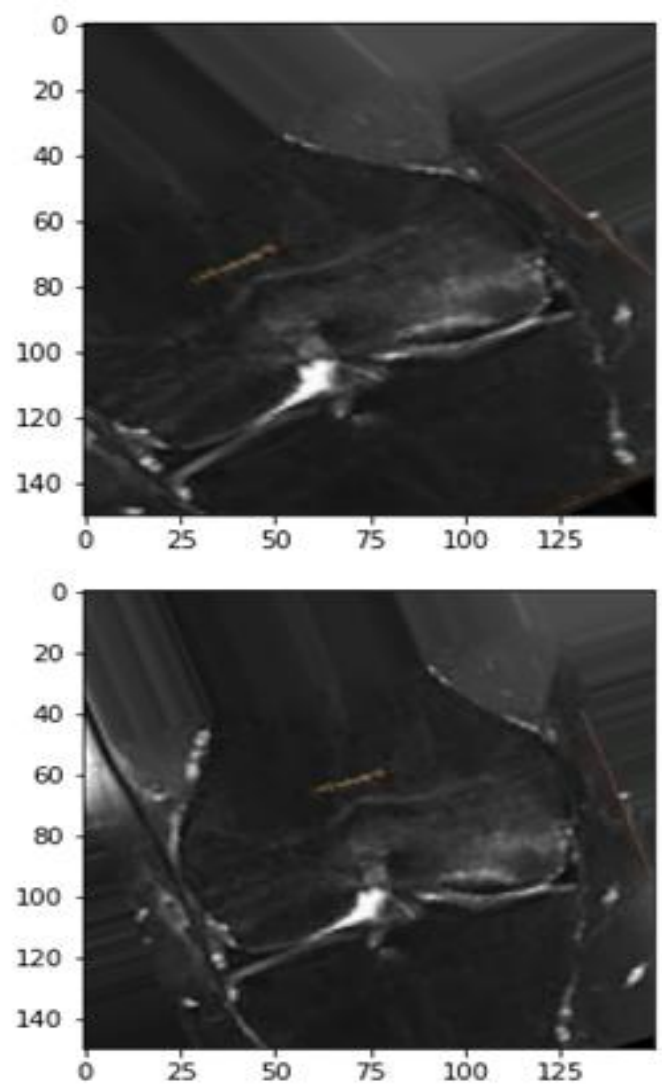

Fig. 6 Deep Obsessive-compulsive disorder (DOCD) Preprocessed MRI images

$$
f(x)_{\text {ParametricLinear }}=\left\{\begin{array}{cc}
x, & \text { if } x>0 \\
a x, & x \leq 0
\end{array}\right\}
$$

b. Activation Function Fully Connected Layer: In a CNN design, this level is mainly found at the very end. Each neuron in this layer is connected to all neurons in the previous layer using the Fully Connected (FC) approach. It is used as a CNN classifier. It describes the fundamental process as a standard
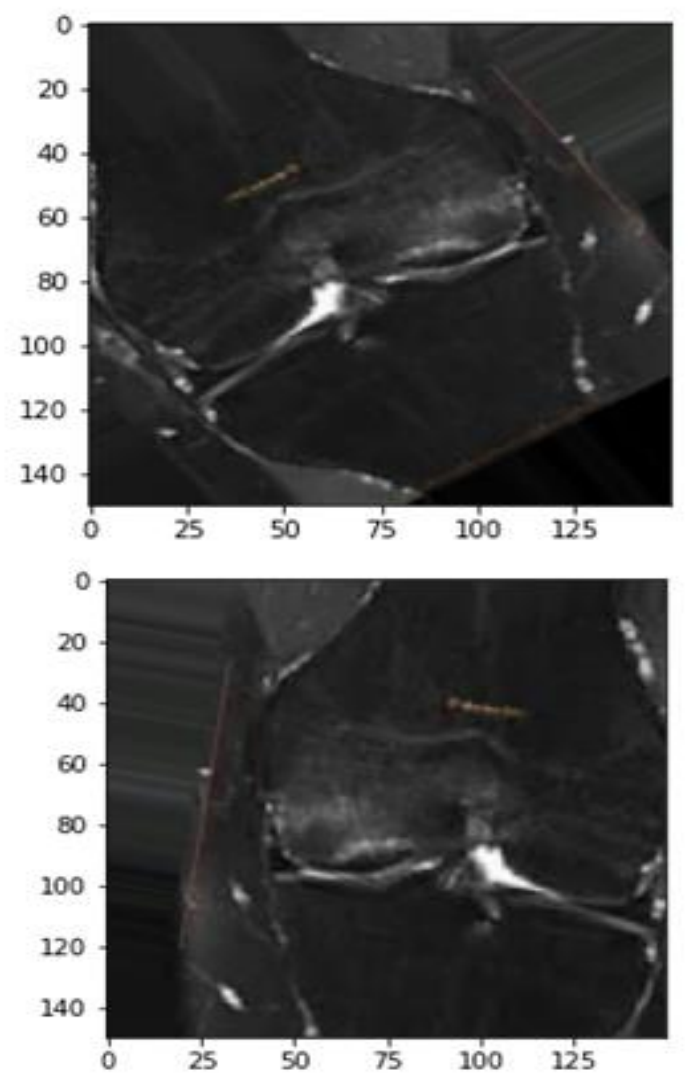


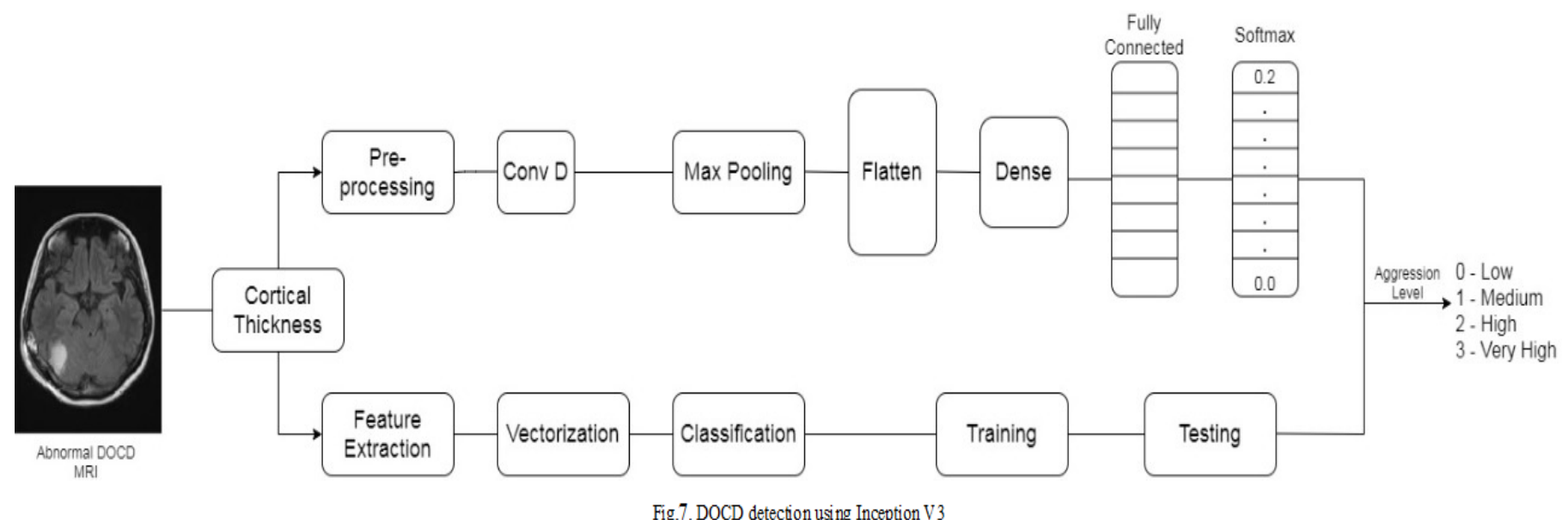

multiple-layer perceptron neural network as a feed-forward ANN. The preceding pooling or convolutional layer provides an input to the FC layer. The feature maps are compressed to form this input, which appears in the form of a vector. The FC layer output reflects the final CNN output, as shown in Figure 4.

The final classification is produced by the output layer, which is the last layer of the CNN design. In the CNN model, the expected error across the training samples is calculated using various loss functions in the output layer. The disparity between the observed and forecast output is revealed by this inaccuracy. The CNN learning procedure will then be used to optimize it. The loss function, on the other hand, uses two parameters to determine the error. The estimated output of $\mathrm{CNN}$ is the first parameter (also known as the prediction). The real output is the second argument (also known as the label).

\section{Inception V3 learning}

Transfer learning is extracting one of the layers from a pretrained model and using it as the input layer to a succession of dense layers. This pre-trained model is typically developed by organizations or businesses with significantly more computational and financial resources. VGG, Inception, and ResNet are some of the most popular image recognition training models. We can then set the parameters of the pretrained model to be non-trainable, and only optimize the parameters of the subsequent dense layers during training using this newly constructed model.

Generally, Machine-learning problems can be solved by Inception V3. The Inception model is a better choice for image classifications for particular images from the deep layer. As depicted in Figure 5, Google announced Inception, a convolutional neural network architecture that won the ImageNet Large Scale Visual Recognition Challenge 2014.

\section{Data Description}

Here the dataset description is based on pattern identification to classify which can be predicted based on the decoded types. The Neuro Imaging dataset can be of data format as Magnetic resonance imaging (MRI) images. Each large-scale pattern from the affected region that are processed has to follow the three parts such as training procedure, testing procedure and validation to understand the severity of Deep Obsessivecompulsive disorder (DOCD). Proposed systems using the underlying brain images can find the stages such as advanced stage, medium stage and initial stage to treat the person in one training destination. On the other hand, normal MRI images of the brain are also trained as well to check and perform classification based on labeled position. The dataset used for the study is from Brain imaging Data Structure-BIDS. The MRI images is stored in OpenNeuro, BIDs database.

Figure.6 represents the sample images of MRI suggesting the presence of DOCD in children with the nucleus structured along with the features as lenticular nuclei. The subject level is based on multi-site images which are mostly generalized from the clinical data sets that are observed from children. Samples are collected from children who are less than 18 years are not diagnosed completely. From the input MRI, T1-weighted brains are initially applied with CNN technique from the base input layer as data acquisition. Analysis is performed from variation of features such as cortical thickness and region of interest(ROI) along with the intracranial depth are classified for training and testing process.

\section{PROPOSED SYSTEM}

Figure.7 represents the overall architecture of the defined deep obsessive compulsive disorder using Transfer learning along with Image classifier Inception V3 which is comparatively better than VGGNET. Refactoring the images based on the process that are given in the descriptive label have a clear view in appropriate results with high performance and low rate. This approach is based on the data to visualize the image classifier and is also based on the analysis where normal brain features are trained to understand the patterns on each layer. This took several number of depth layers for normalizing the image as a random variable.

Transfer learning is one of the smart pre-trained model for image classification that can work with Convolutional Neural Network (CNN) for predicting aggression level for a better accuracy. This transfer learning usually extracts one important features from the individual layers, which becomes the input for the next stage of computation in the dense layer. Using MRI images, transfer learning is applied to learn the unique 
features that can observe the obsession aggression level and training undertaken accordingly. Using Inception-V3 model, the system can compute in a very less time to make the performance level high. The importing of the proposed model along with the packages such as keras, tensorflow for multiple layer perceptrons. This fully connected layer maintains the weight in the features. As the dense layer reduces the number of working layers, the lowest layer can treat the MRI images and retrain the model which can improve the performance by avoiding overfitting issues.

Tensorflow and Keras are used to generate the input from the model using the DOCD aggression problem. The categorical variables are validated about $30 \%$ to get accurate results from random changes. Image classifier results in a powerful performance using Inception V3 where the previous learning feature knowledge is given to the current layer which is using neural network model. A comparison of existing analysis with the ResNet is done. The previously trained knowledge is transferred to current labeled features. The training data is in an ordered manner to find the accuracy from the abnormal brain MRI as classes, which are mentioned as training information. Whenever the unlabeled MRI features are not built according to the multi-layer perceptron, assigned weight and bias visualize an unfinished task. The study and its performance is from the beginning point where the general framework transfers learning with the principles of Inception V3 model. Large-scale and various Convolutional layers with the max pooling filter that are represented in Figure.8a and $8 \mathrm{~b}$ are involved with the input that are with the trained dataset for normal brain and DOCD MRI images. These layers are available altogether from a block that are repeated many times after the filter $3 * 3$ has updated the dense layer frequently. The multi perceptron layer implies the fully connected layer with the network as 32,64 for the blocks of the proposed model. In addition, the length and width from the block are arranged with the padding layer to finalize the height and width with the 128 and 256 that are identified from the dense layer which are mapped with the features that are raised from the MRI input images.

\section{Algorithm 1}

Transfer learning : TL-INCEPTION V3 -CNN

Start

Task 1 - Input : MRI Input data $\{\mathrm{F} 1, F 2, \ldots F p h\}$

Task 2 - Input:

Image feature analysis based on aggression modality level $\{(\mathrm{w} 1, \mathrm{~h} 1),(\mathrm{w} 2, \mathrm{~h} 2) \ldots(\mathrm{wm}, \mathrm{hm})\}$ Q)

Output - DOCD - TL-INCEPTION V\# model (weight, bias,

\# transfer learning initial layer

for $n-1: L$ patterns of MRI

Min E(weight, bias)
Stop

\# Increasing performance and low error rate

Match the credentials based on identified subjects

Applying weight,shape and bias

classifier_activation =forward pass

feature_4D (Dense layer)

Aug_img = reduce error rate (horizontal flipping)

frame $=$ dim $($ aug_img(255)

reduce false training $=$ overfitting $($ high error rate - testing set)

The proposed model consists of two parts for classification and training such as phase 1 and phase 2 .

Phase 1 - Training dataset using Inception V3 -Transfer learning (TL) framework by learning the image from the gathered dense layer.

Phase 2 - To reduce time and increase performance rate as maximum using transfer learning where less data loss occurs during fully connected process from the pre-trained model.

To understand the pattern of DOCD MRI images which are unstable such that unsupervised manner cannot be reliable like normal brain features. Pathogenesis of DCOD helps in analyzing the report of modality ranges which are abnormal than the normal brain. We identified the selective subjects from 150 subjects with DOCD underwent patient data. As behavioral changes are one of the major differences in children, certain independent variables are used for obtaining the accuracy level from the aggression level. In the study the variables defined were the dependent variable and the aggressive personality. In addition, the independent variables could be the influence of parents and their relationship. For the purpose of study to measure the changes and measure the aggression level we could compare the emotional relationship with parents and also associate the a child's behavior based on how parents treat them in different situation such as at home or outside or how parents react with then when they are angry or pleasant etc. In addition, a variable could also be determined to measure the gender differences of aggression level based on the parents and children. Changes in them can be classified and detected using transfer learning algorithms. Using Convolutional Neural Networks (CNN), input such as MRI (Magnetic resonance Imaging) is used for experimenting the variations in behavior with the high dimension that are analyzed from the image dataset. Using Inception V3-Transfer Learning algorithms that are defined in Table.1, CNN generalization of misophonia level can be statistically analyzed to avoid overfitting problems. The aggression level can be predicted by data augmentation with better accuracy and a low error rate than the existing systems. 


\section{RESULTS AND DISCUSSION}

Using the MRI dataset, the aggression level prediction uses the severity level as low, medium and high from the influence of independent variables on classification and its accuracy level based on performance. As the disease duration is observed in a short range of years in children, severity is obtained based on the AUC and its probability. This is the aim of obsession and disorder in pharmacologic approach. Using the proposed model image batch based on the feature extraction is done which converts the size of blocks. The convolution layer as shown in figure. 1 needs to be freeze to set the training weight standard based on the Inception V3 classifier. When the batch normalization returns to the mode of minimized mean and variance curve, then the statistical analysis is fine tuned. To predict the severity from the standard blocks that have element vector match along with max pooling layer, supports finding the average in multi-prediction from the MRI image.

Using the binary cross entropy the argument are processed along with the metrics accuracy. When the variation from training image generation and validation are rescaled with 1.2 of 255 from the training images which are of among 20 batches from the validation directory target size and batch size are identified with binary class mode. Probability ratio based on feature mapping is visualized with each filter that are displayed with the grid according to the size difference. Layer wise training knowledge and transfer learning using CNN are depicted in Figure.9.

The main nerve system of the brain is the posterior cingulated cortex (PCC) where all the instructions are passed by as an important communication partner. At the same time, memory saving features through MRI is recognized. As the main features for our model we consider the PCC, Cortical thickness (CT), surface area (SA) to fix the abnormality and aggression level. From our gathered datasets of limited patients MRI results the variation using categorical features is shown in Table 1. along with the training and testing results. Figure. 10 depicts the accuracy variation of each epoch that is monitored and compared with the existing results. Based on shear range and nearest flip mode the results show high accuracy. As the input is fetched from preprocessed MRI dataset images, the Convolution layer, max pooling, Flatten, dropout reduces the blocks and connects the fully connected layer based on the softmax layer filtered data to identify the severity that changes from the normal brain images. From the network information Table 1, the multi perceptron functions along with factors such as generalized methods and sum, mean and standard deviation gives the implemented results. Table.1 determines the results based the error rate decreased and loss validation based on the factors assigned for each dependent variables.

To identify the affected part from cortical thickness and surface area that are curved from the MRI regions Table 2 provides the proposed model summary, which increases the performance and reduces the low error rates according to the image classification produced results.
Few studies show that less volume has failed in fixing the Region of interest (ROI) and shows that the abnormality and its modality changes are imperfect. A comparison probability and the processed images are analyzed for fixing the matrix level that fits the filter to the proposed model. The segmented brain and the tissues that are identified from the mean square rate difference shown in Figure.11 support corrections that can change the classifier to predict the right cortical surfaces. Table 2. shows the hidden unit and the criteria based on the known results that are compared between training and testing value as a model summary.

Table 1. Determined by the testing data criterion: The "best" number of hidden units is the one that yields the smallest error in the testing data.

\begin{tabular}{|c|c|c|c|}
\hline \multicolumn{4}{|c|}{ Network Information } \\
\hline \multirow{2}{*}{$\begin{array}{l}\text { Input } \\
\text { Layer }\end{array}$} & Factors & 1 & Algorithm \\
\hline & \multicolumn{2}{|l|}{ Number of Units } & 2 \\
\hline \multirow{2}{*}{$\begin{array}{l}\text { Hidden } \\
\text { Layer }\end{array}$} & \multicolumn{2}{|l|}{ Number of Units } & $7^{\mathrm{a}}$ \\
\hline & \multicolumn{2}{|l|}{ Activation Function } & Softmax \\
\hline \multirow{5}{*}{$\begin{array}{l}\text { Output } \\
\text { Layer }\end{array}$} & Dependent Variables & 1 & Accuracy \\
\hline & \multicolumn{2}{|l|}{ Number of Units } & 1 \\
\hline & \multicolumn{2}{|c|}{$\begin{array}{l}\text { Rescaling Method for Scale } \\
\text { Dependents }\end{array}$} & Standardized \\
\hline & \multicolumn{2}{|l|}{ Activation Function } & Identity \\
\hline & \multicolumn{2}{|l|}{ Error Function } & Sum of Squares \\
\hline
\end{tabular}

Table 2. The number of hidden units is determined by the testing data criterion: The "best" number of hidden units is the one that yields the smallest error in the testing data.

\begin{tabular}{|l|l|l|}
\hline \multicolumn{2}{|c|}{ Model Summary } & 28.914 \\
\hline Training & Sum of Squares Error & 0.650 \\
\hline & Relative Error & $0.00: 00.03$ \\
\hline \multirow{2}{*}{ Testing } & Training Time & $6.091^{\mathrm{a}}$ \\
\hline & Sum of Squares Error & \\
\hline & Relative Error & 0.527 \\
\hline
\end{tabular}


INTERNATIONAL JOURNAL OF BIOLOGY AND BIOMEDICAL ENGINEERING

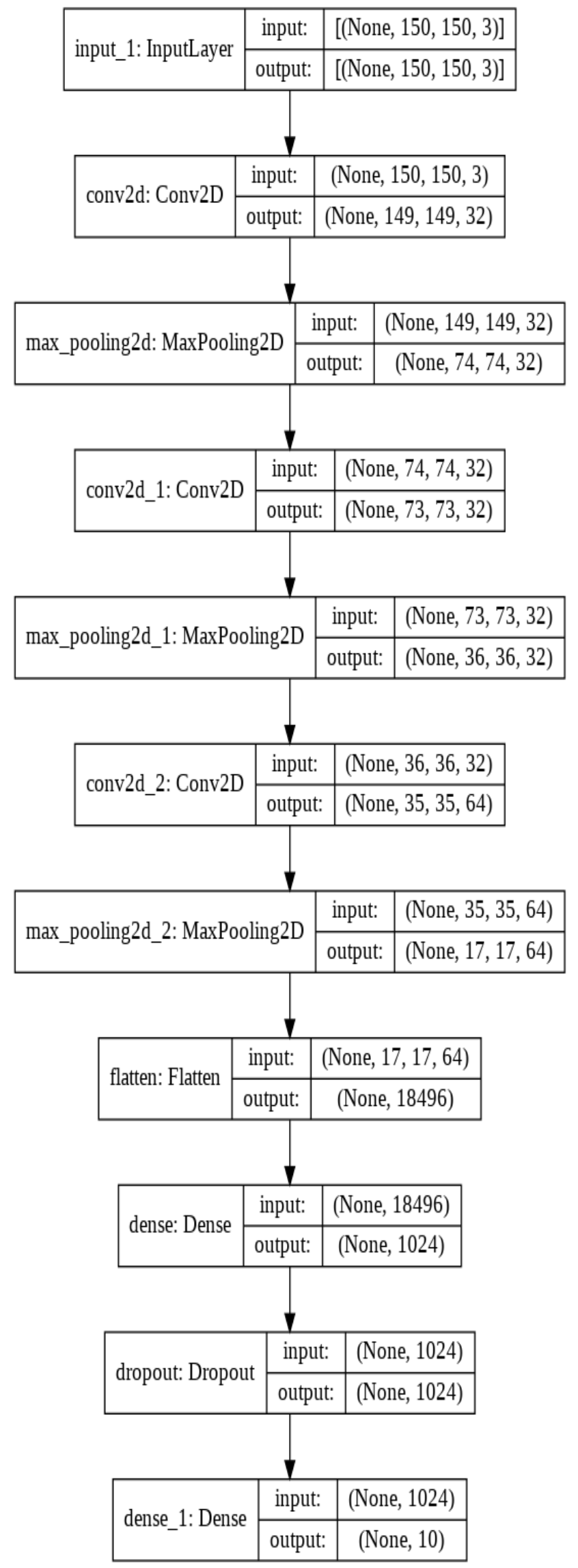

Fig.8(a). CNN workflow from pre-trained model using image classifier

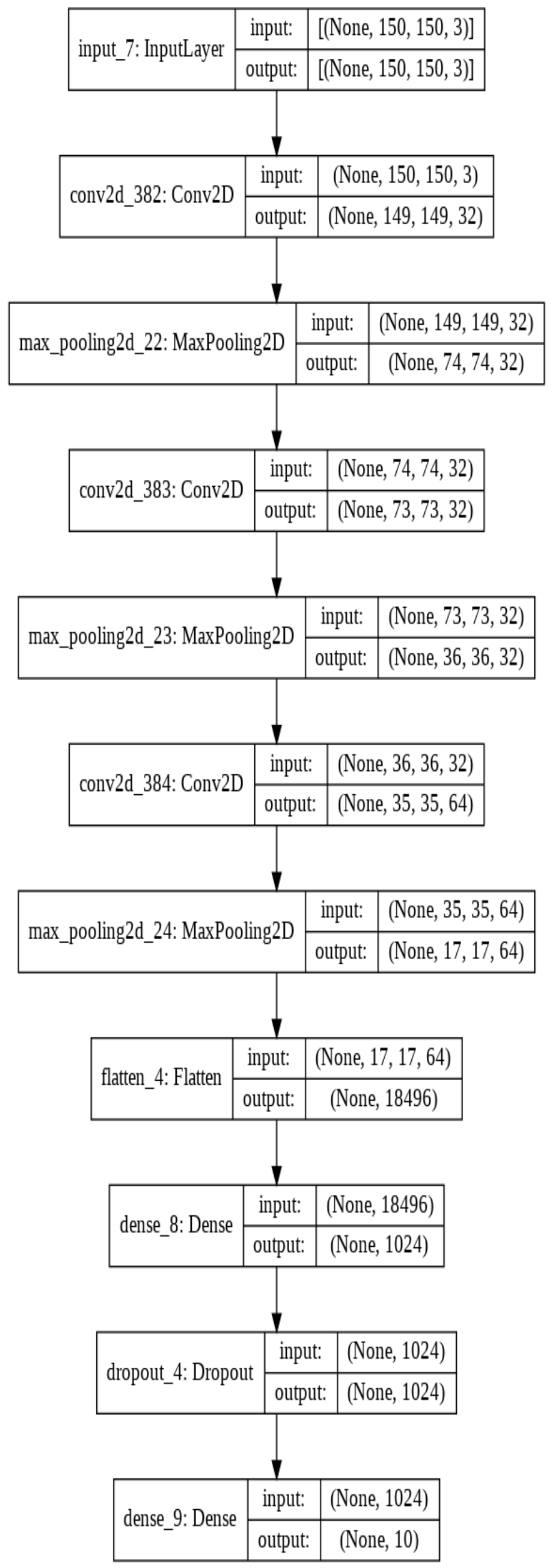

Fig.8(b). CNN workflow from pre-trained model using image classifier 
INTERNATIONAL JOURNAL OF BIOLOGY AND BIOMEDICAL ENGINEERING
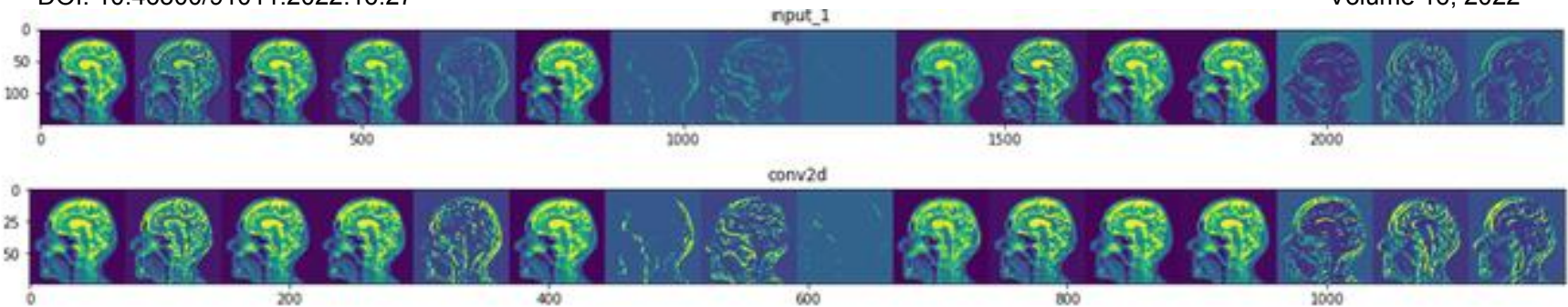

$\max$ pooling 20
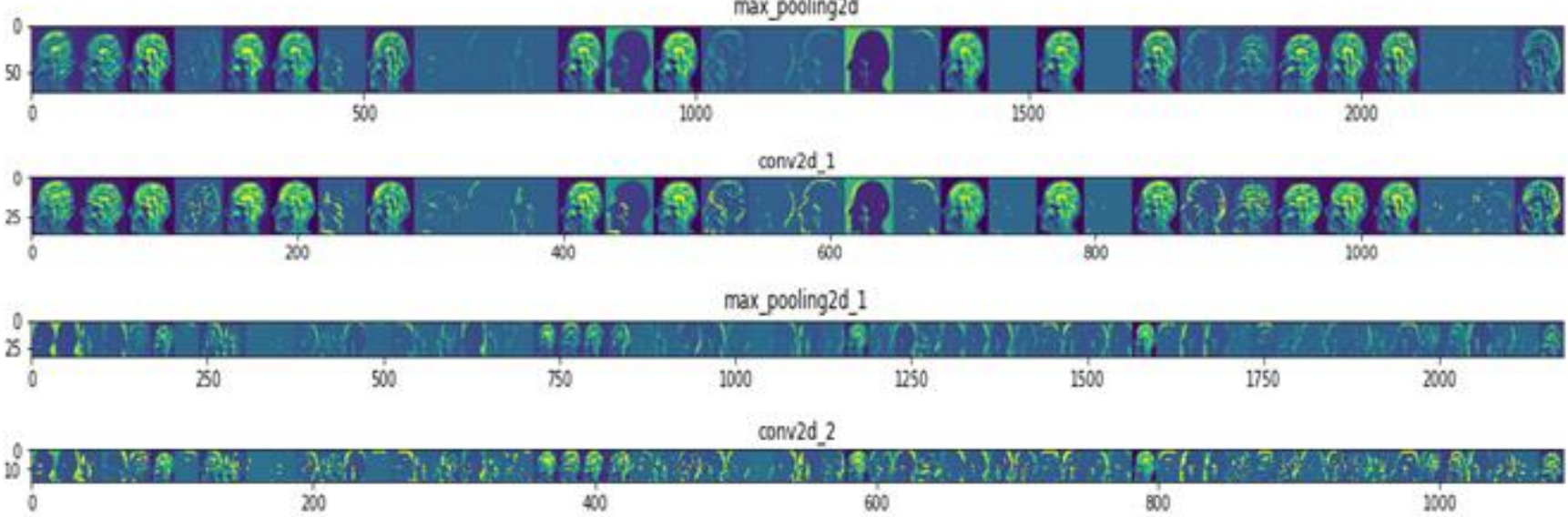

Fig.9. Display grid using Transfer leaming of DOCD

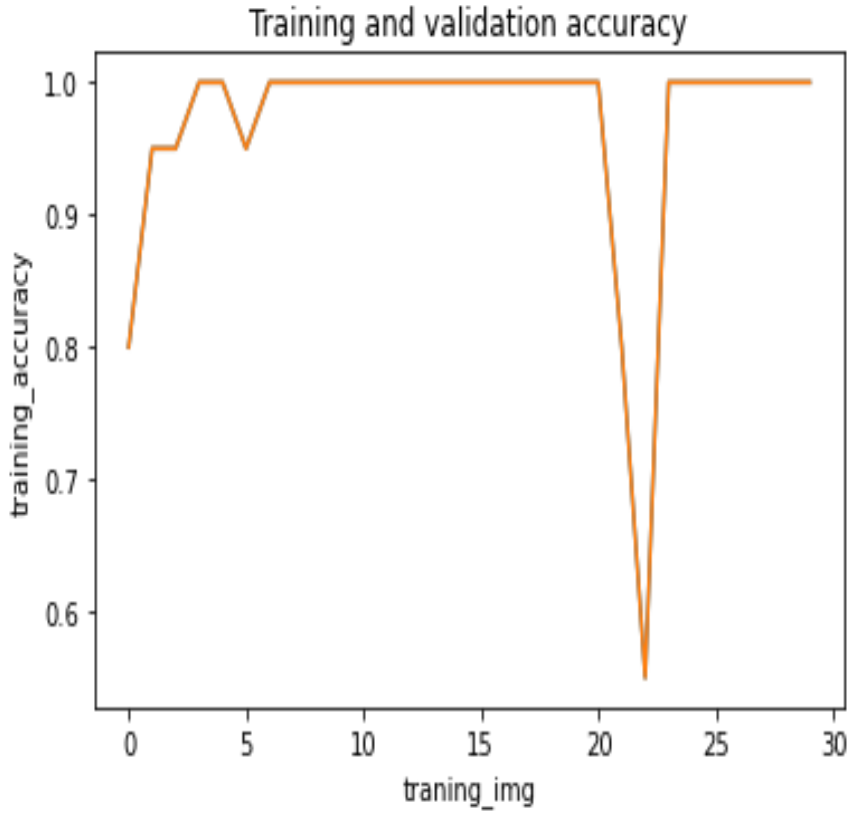

Fig. 10(a). Training and validation accuracy \& Validation loss rate

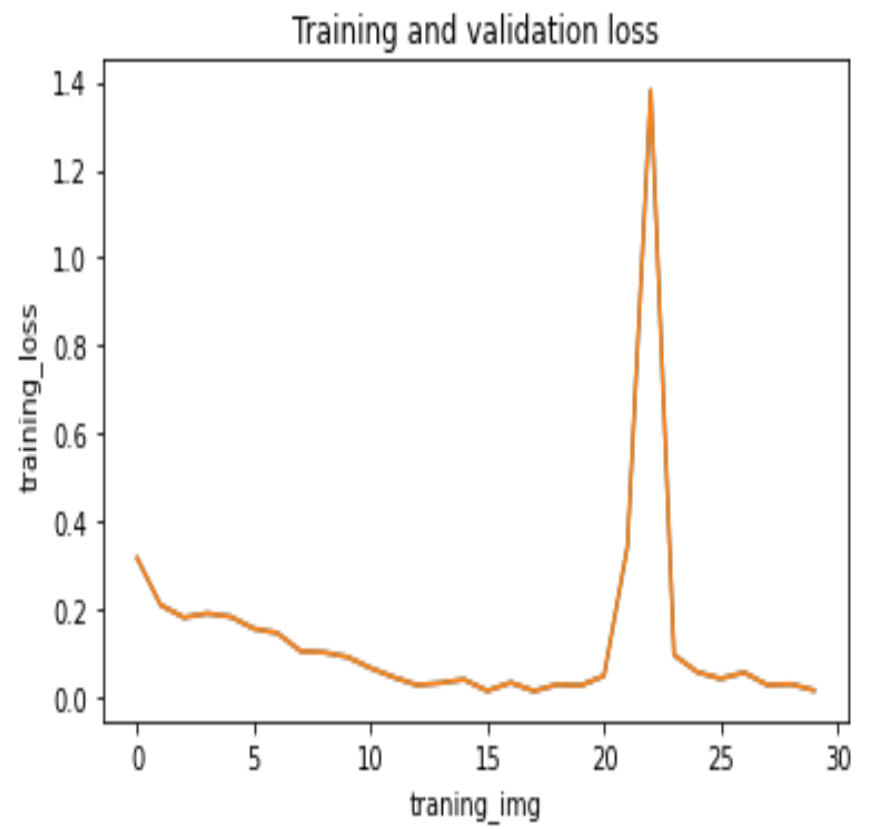

Fig. 10(b). Training and validation accuracy \& Validation loss rate 


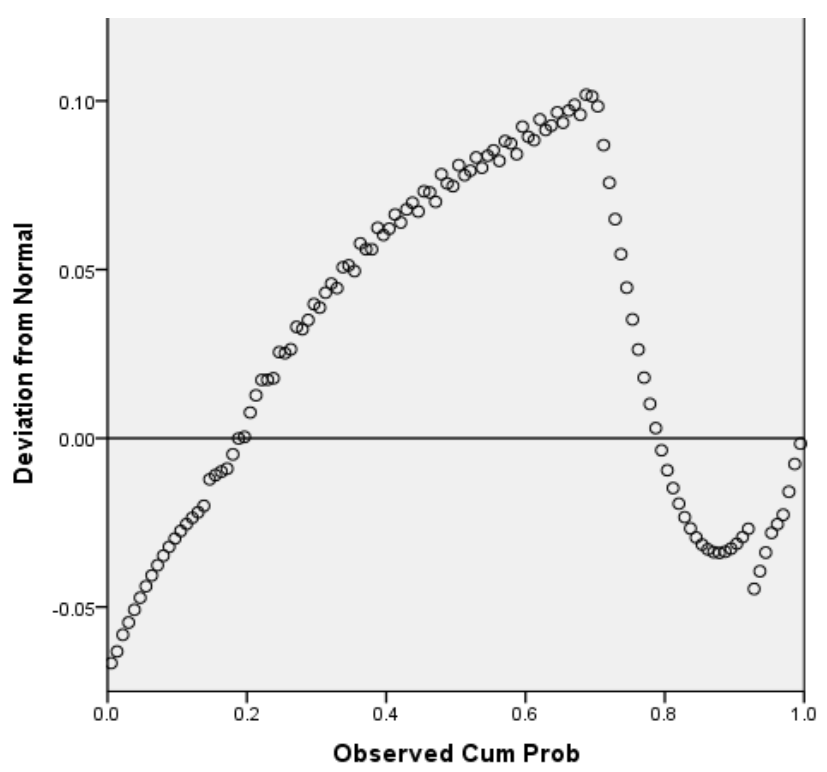

Fig 11. Dependent Variable: Accuracy

\section{CONCLUSION}

Deep obsessive-compulsive disorder has been a serious issue in children in this era. The level to which it has caused aggression in children has been varying and depending on several factors. This depends on the activities they are involved in and the tolls and time they are using them. The use of mobiles, tablets and gaming units such as play stations have been found to be a critical factor in keeping the children to themselves. Disturbing behaviours such as sitting themselves and talking, selfishness are some of the visible symptoms of DOCD. The DOCD disorder is linked to the brain and MRI scans reveal a lot of similarity linked to many children having it. An approach to detect aggressive level in children at an earlier stage needs to be given prior importance. Artificial intelligence methods such as deep learning has been used early to study the disorder and the level of aggression children may be displaying. However, CNN poses several disadvantages of clarity in classification. In addition, when employing CNN it has been observed that the images get classified with various different positions. Moreover, the performance becomes a questionable factor when $\mathrm{CNN}$ is used alone. The method proposed in this research involves a hybrid of CNN and Inception-V3 transfer learning to predict the level of aggression in children with DOCD. Initially data is collected from children with abnormal behaviours and inception V3 transfer learning $\mathrm{CNN}$ is utilized for the analysis process. Accuracy and error rate are analysed for the proposed work to prove the efficacy of the system. Through a gradual increase of disorder in children, using the dataset identified the depression level of DOCD. Using the proposed model Inception-V3 transfer learning $\mathrm{CNN}$, the aggression levels are predicted with a lower rate of 0.3 in training and testing as 0.527 that is better than the existing $\mathrm{CNN}$ model.

\section{References}

[1] Rajan Kashyap, Goi Khia Eng, Sagarika Bhattacharjee, Bhanu Gupta, Roger Ho, Cyrus S. H. Ho, Melvyn Zhang, Rathi Mahendran, Kang Sim \& S. H. Annabel Chen, Individual-fMRI-approaches reveal cerebellum and visual communities to be functionally connected in obsessive compulsive disorder, Scientific Reports, 11, Article number: 1354 (2021)

[2] Luka C Liebrand, Paul Zhutovsky, Eva K Tolmeijer, Ilse Graat, Nienke Vulink, Pelle de Koning, Martijn Figee, P Richard Schuurman, Pepijn van den Munckhof, Matthan W A Caan, Damiaan Denys, Guido A van Wingen ${ }^{-}$Deep brain stimulation response in obsessive-compulsive disorder is associated with preoperative nucleus accumbens volume, NeuroImage: Clinical, Volume 30, 2021, 102640.

[3] Lekai Luo, Qian Li, Wanfang You, Yuxia Wang, Wanjie Tang, Bin Li, Yanchun Yang, John A. Sweeney, Fei Li, Qiyong Gong, Altered brain functional network dynamics in obsessive-compulsive disorder, Wiley Online Library, ,February 2021

[4] Manuel Mattheisen, Jack F. Samuels, Ying Wang, Benjamin D. Greenberg, Abby J. Fyer, James T. McCracken, Daniel A. Geller, et alGenome-wide association study in obsessive-compulsive disorder: Results from the ocgas, Mol. Psychiatry 20 (3) (2015) 337.

[5] Eric Burguiere, Patricia Monteiro, Luc Mallet, Guoping Feng, Ann M. Graybiel, Striatal circuits, habits, and implications for obsessive-compulsive disorder, Curr. Opin. Neurobiol. 30 (2015) 59-65.

[6] I. Angelakis, P. Gooding, N. Tarrier, M. Panagioti, Suicidality in obsessive compulsive disorder (OCD) a systematic review and meta-analysis, Clin. Psychol. Rev. 39 (2015) 1-15.

[7] A. Sidorchuk, R. Kuja-Halkola, B. Runeson, P. Lichtenstein, H. Larsson, C. Rück, et al., Genetic and environmental sources of familial coaggregation of obsessive compulsive disorder and suicidal behavior: a population-based birth cohort and family study, Mol. Psychiatry 1 (2019).

[8] American Psychiatric Association, et al., Diagnostic and statistical manual of mental disorders, BMC Med. 17 (2013) 133-137.

[9] Lorrin M. Koran, Gregory L. Hanna, Eric Hollander, Gerald Nestadt, Helen Blair Simpson, American Psychiatric Association, et al., Practice guideline for the treatment of patients with obsessive-compulsive disorder, Am. J. Psychiatry 164 (Suppl. 7) (2007) 5-53.

[10] Jon E. Grant, Obsessive-compulsive disorder, New Engl. J. Med. 371 (7) (2014) 646-653.

[11]Eva M. Cybulska, Obsessive-compulsive disorder, the brain and electroconvulsive therapy, Br. J. Hosp. Med. 67 (2) (2006) 77-82, 2005.

[12] Borjanka Batinic, Duisin Dragana, PT610. aripiprazole augmentation in patients with Ocd partially responsive to 
Ssri, Int. J. Neuropsychopharmacol. 19 (Suppl. 1) (2016) 24.

[13] Tomoya Takeda, Satsuki Sumitani, Sayo Hamatani, Yosuke Yokose, Megumi Shikata, Tetsuro Ohmori, Prefrontal cortex activation during neuropsychological tasks might predict response to pharmacotherapy in patients with obsessive-compulsive disorder, Neuropsychiatric Dis. Treat. 13 (577) (2017).

[14] Mohammadreza Shalbafan, Farzaneh Malekpour, Borna Tadayon Najafabadi, Kiandokht Ghamari, Seyed-Ali Dastgheib, Arash Mowla, Elham Shirazi, Mehrdad Eftekhar Ardebili, Maryam Ghazizadeh-Hashemi, Shahin Akhondzadeh, Fluvoxamine combination therapy with tropisetron for obsessive-compulsive disorder patients: A placebo-controlled, randomized clinical trial, J. Psychopharmacol. (2019) 0269881119878177.

[15] Mehdi Sayyah, Fakher Rahim, Pharmacotherapy for treatment-respondent vs. refractory obsessive-compulsive disorder in children and adults: Strategies, meta-analyses and clinical guidelines, Arch. Psychiatry Psychother. 3 (2018) 42-54.

[16] A. Esteva, A. Robicquet, B. Ramsundar, V. Kuleshov, M. DePristo, K. Chou, et al., A guide to deep learning in healthcare, Nat. Med. 25 (1) (2019) 24-29.

[17] N.S. Abul-Husn, E.E. Kenny, Personalized medicine and the power of electronic health records, Cell 177 (1) (2019) 58-69.

[18] Sigal Zilcha-Mano, Steven P. Roose, Patrick J. Brown, Bret R. Rutherford, A machine learning approach to identifying placebo responders in late-life depression trials, Am. J. Geriat. Psychiatry 26 (6) (2018) 669-677.

[19] Shani Stern, Sara Linker, Krishna C. Vadodaria, Maria C. Marchetto, Fred H. Gage, Prediction of response to drug therapy in psychiatric disorders, Open Biol. 8 (5) (2018) 180031.

[20]Eugene Lin, Hsien-Yuan Lane, Machine learning and systems genomics approaches for multi-omics data, Biomarker Res. 5 (1) (2017).

[21] Farnaz Zamani Esfahlani, Katherine Visser, Gregory P. Strauss, Hiroki Sayama, A network-based classification framework for predicting treatment response of Schizophrenia patients, Expert Syst. Appl. 109 (2018) 152-161.

[22]F.S. Collins, M. Morgan, A. Patrinos, The Human Genome Project: lessons from large-scale biology, Science 300 (5617) (2003) 286-290.

[23] Eugene Lin, Po-Hsiu Kuo, Yu-Li Liu, Younger W.-Y. Yu, Albert C. Yang, Shih- Jen Tsai, A deep learning approach for predicting antidepressant response in major depression using clinical and genetic biomarkers, Front. Psychiatry 9 (290) (2018).

[24] Eugene Lin, Yuchi Hwang, Shu-Ching Wang, Z. John Gu, Ellson Y. Chen, An artificial neural network approach to the drug efficacy of interferon treatments, 2006.
[25] Eugene Lin, Yuchi Hwang, A support vector machine approach to assess drug efficacy of interferon- $\alpha$ and ribavirin combination therapy, Mol. Diagn. Ther. 12 (4) (2008) 219-223.

[26] Adam Mourad Chekroud, Ryan Joseph Zotti, Zarrar Shehzad, Ralitza Gueorguieva, Marcia K. Johnson, Madhukar H. Trivedi, Tyrone D. Cannon, John Harrison Krystal, Philip Robert Corlett, Cross-trial prediction of treatment outcome in depression: A machine learning approach, Lancet Psychiatry 3 (3) (2016) 243-250.

[27] Andrew F. Leuchter, Ian A. Cook, Steven P. Hamilton, Katherine L. Narr, Arthur Toga, Aimee M. Hunter, Kym Faull, et al., Biomarkers to predict antidepressant response, Curr. Psychiatry Rep. 12 (6) (2010) 553-562.

[28] Andrew F. Leuchter, Ian A. Cook, Lauren B. Marangell, William S. Gilmer, Karl S. Burgoyne, Robert H. Howland, Madhukar H. Trivedi, et al., Comparative effectiveness of biomarkers and clinical indicators for predicting outcomes of Ssri treatment in major depressive disorder: Results of the brite-Md study, Psychiatry Res. 169 (2) (2009) 124-131.

[29] Yifei Chen, Yi Li, Rajiv Narayan, Aravind Subramanian, Xiaohui Xie, Gene expression inference with deep learning, Bioinformatics 32 (12) (2016) 1832-1839.

[30] Eugene Lin, Shih-Jen Tsai, Genome-wide microarray analysis of gene expression profiling in major depression and antidepressant therapy, Prog. NeuroPsychopharmacol. Biol. Psychiatry 64 (2016) 334-340.

[31] Madanan, M., Venugopal, A., Designing a Hybrid Model Using HSIC Lasso Feature Selection and Adaboost Classifier to Classify Image Data in Biomedicine. International Transaction Journal of Engineering, Management, \& Applied Sciences \& Technologies, 12(1), 12A1G, 1-14(2021)

[32] Madanan.M,Venugopal.A,Velayudhan.C.N.,Applying an Optimal Feature Ranking and Selection Algorithm and Random Forest Classifier Algorithm along with K-Fold Cross Validation for Classification of Blood Cancer Cells,European Journal of Molecular \& Clinical Medicine,Vol 07,Issue 11(2020).

Mr.Mukesh Madanan is a Senior Lecturer of Computer Science at Dhofar University, Oman. He completed his Bachelors in Technology in Computer Science and Engineering from M.G. University, India and went on to complete his M.Sc.in Software Engineering from the University Of Portsmouth, UK. He is currently pursuing $\mathrm{PhD}$ in Information \& Communication Technology from UNITEN, Malaysia. His areas of research include Machine Learning, Deep Learning, Robotics, Software Methodologies, IoT and Computer Networks.

Dr. Biju Theruvil Sayed is a higher educational academician with a total experience of $25+$ years in the field of Computer Science/Computer Engineering/Management Information 
Systems/Information Technology. As of the current date, he has been involved in teaching and learning processes for an approximate of $12000+$ candidates. He is currently associated as a Chairperson and Assistant Professor in Computer Science at Dhofar University in Salalah, Oman. In addition, he is a Certified Training Professional and his current research area and interests are in Education Management, Education Assessment, Knowledge Management Systems, Expert Systems.

\section{Contribution of Individual Authors to the Creation} of a Scientific Article (Ghostwriting Policy)

Mukesh Madanan carried out the implementation of algorithm, simulation and the optimization of the entire process.

Biju T. Sayed has done the literature review and data collection.

\section{Creative Commons Attribution License $\mathbf{4 . 0}$}

(Attribution 4.0 International, CC BY 4.0)

This article is published under the terms of the Creative Commons Attribution License 4.0

https://creativecommons.org/licenses/by/4.0/deed.en_US 